\title{
Robustness of a discrete-time predictor-based controller for time-varying measurement delay
}

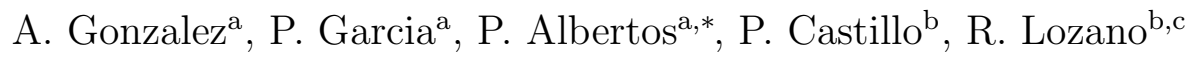 \\ ${ }^{a}$ Instituto Automática e Informática Industrial, \\ Universitat Politècnica de València, E-46022 Valencia, Spain \\ ${ }^{b}$ Heudiasyc UMR 6599 - UTC. B.P. 20529, Compiègne, France \\ ${ }^{c}$ UMI 3175 LAFMIA - CINVESTAV Mexico
}

\begin{abstract}
A predictor-based controller for time-varying delay systems is presented in this paper and its robustness properties for different uncertainties are analyzed. First, a time-varying delay dependent stability condition is expressed in terms of LMIs. Then, uncertainties in the knowledge of all plant-model parameters are considered and the resulting closed-loop system is shown to be robust with respect to these uncertainties. A significant improvement with respect to the same control strategy without predictor is achieved. The scheme is applicable to open-loop unstable plants and it has been tested in a real-time application to control the roll angle of a quad-rotor helicopter prototype. The experimental results show good performance and robustness of the proposed scheme even in the presence of long delay uncertainties.
\end{abstract}

Keywords: Time-varying delay, discrete-predictor, digital implementation, linear matrix inequality (LMI), robust stability.

\section{Introduction}

In designing any control system the process behavior imposes some unavoidable performance limitations (see, for instance, Seron et al. (1997)).

\footnotetext{
This work has been partially granted by Conselleria de Educación under PROMETEO project number 2008-088, and CICYT no. DPI2008-06737-C02-01 from Spanish government.

*Corresponding author

${ }^{1}$ Email address: pedro@aii.upv.es (P. Albertos)
} 
This is clearly the case when dealing with systems with time delays, see for example Normey-Rico and Camacho (2007); Normey-Rico et al. (2009); Ingimundarson and Hagglund (2001) and the references therein. The Smith Predfictor (SP), Smith (1957), and the Finite Spectrum Assignment (FSA), Manitius and Olbrot (1979), may be considered as the main control methods for linear processes with time delay in either the input or the output (see also Richard (2003)). A careful analysis of these methods and their modifications shows that they all use, in an implicit or explicit manner, a prediction of the state in order to achieve the control of the system. The more appealing characteristic of a predictor-based control is that, for a delayed plant without modelling error, the achieved performance approaches that of a delay-free system, Yue and Han (2005), Normey-Rico and Camacho (2008), Guzman et al. (2008).

As explained in Palmor (1996), the use of an explicit unstable prediction model in the SP approach determines the internal instability of the closedloop system. Palmor also suggested how to implement the control law using the so-called integral form. In Manitius and Olbrot (1979), this approach was also introduced in the framework of spectrum assignment with distributed delays. However, as shown in Mondié et al. (2002), the implementation of this control approach on a digital computer may result in an unstable behavior.

Nowadays, almost any control system application is implemented by using a computer, Astrom and Wittenmark (1997). Thus, it is worth to analyze the effect of the delays in sampled data systems. On the other hand, it is wellknown that Discrete-Time (DT) delayed systems can be transformed into a delay-free system by using state augmentation techniques. However, in many cases, such delays are too large and time-varying, Yue and Han (2005), Pan et al. (2006). In such cases, conventional state augmentation technique is usually not applicable, Gao and Chen (2007). These considerations motivate the study of time-varying DT systems, Zhong (2004), Boukas (2006). Different control strategies have been proposed, such as a static state feedback Du et al. (2008); Guo and Li (2009); Meng et al. (2010) and Guangdeng et al. (2009) or a dynamic controller Liu et al. (2006); He et al. (2008).

In Lozano et al. (2004), a discrete-time controller for Continuous-Time (CT) plants with time delay is proposed and the closed-loop stability is proved. The proposed robust stability proof allows positive or negative variations in the delay but they must be bounded by the size of the sampling 
period. In Garcia et al. (2006), the previous results were enlarged when the delay uncertainty is larger than the sampling period but, in both papers, time-varying delay variations smaller than the sampling period are only considered. Recently in Garcia et al. (2010), this framework was improved in order to analyze time-varying delays greater than the sampling period, although the LMI constraints developed to check closed-loop stability have been shown to be very conservative.

The objective of this paper is to analyze robust stability of DT predictorbased state-feedback controllers applied to open-loop unstable systems with time-varying measurement delay, when only delay bounds are assumed to be known. To the best of the authors' knowledge, the robust stability analysis under large but bounded time varying-delays has not been previously reported in the literature.

The paper is organized as follows: the problem statement is introduced in the next section. The stability condition to analyze time-varying delay systems is proposed in Section 3. Section 4 is devoted to present some numerical examples to compare the performance of the predictor-based and the equivalent memoryless control schemes. In section 5, robustness issues are analyzed. In order to validate the proposed control scheme in real time operation, several experiment tests are carried-out in Section 6. Conclusions are drawn in Section 7.

\section{Problem formulation}

Assume the following CT state space representation of the plant

$$
\begin{aligned}
& \dot{x}(t)=A_{c} x(t)+B_{c} u(t) \\
& x(t)=0, \quad t \leq 0
\end{aligned}
$$

where the nominal plant parameter matrices are $A_{c} \in \Re^{n \times n}, B_{c} \in \Re^{n \times m}$. Without loss of generality, and with the aim to simplify the analysis, null initial conditions are assumed.

As depicted in Figure 1, the plant state $x(t)$ is fully accessible but there is an unknown measurement/transmission delay $\tau(t)$. That is

$$
\tilde{x}(t)=x(t-\tau(t))
$$

This delay is assumed to be randomly varying, $\tau(t)$, but bounded by known values. 


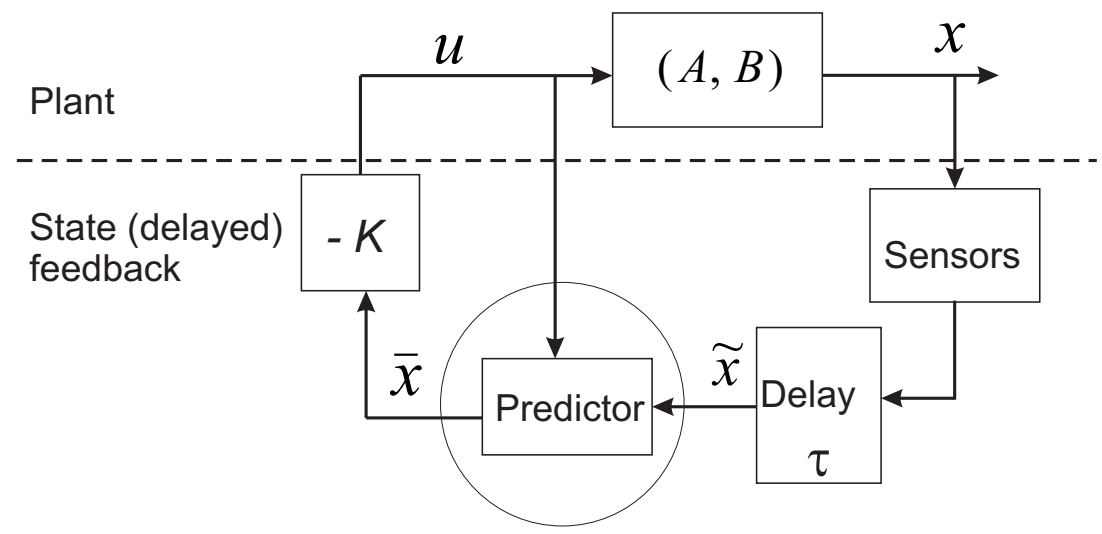

Figure 1: State-feedback control of measurement delayed plants.

Since a computer implementation is intended, the sampling period is defined as $T_{k}=t_{k+1}-t_{k}$, where $t_{k}$ is the sampling time instant, $k \in Z^{+}$, and $\tau\left(t_{k}\right)=\tau_{k}$.

In this section, the following assumptions are made ${ }^{2}$ :

Assumption A1. The sampling period is constant $T_{k}=T$.

Assumption A2. The output delay satisfies $\tau_{k}=d_{k} T$, where $d_{k} \in Z^{+}$and $d_{m} \leq d_{k} \leq d_{M}$, being $d_{m}$ and $d_{M}$ known delay bounds ${ }^{3}$. That means $d_{k}$ is also randomly varying.

Assumption A3. There are no uncertainties in the process model $\left(A_{c}, B_{c}\right)$.

Then, a DT approximation of (1) is given by

$$
\begin{aligned}
x_{k+1} & =A x_{k}+B u_{k} \\
x_{k} & =0, \quad k \leq 0
\end{aligned}
$$

where $A=e^{A_{c} T}, B=\int_{0}^{T} e^{A_{c} \sigma} d \sigma B_{c}$ and $d_{k}$ is the discrete-time delay, defined in Assumption A.2.

A state feedback law is assumed

$$
u_{k}=-K x_{k}
$$

\footnotetext{
${ }^{2}$ These assumptions will be removed in Section 5 .

${ }^{3}$ In general, $d_{m}$ is known and fixed whereas $d_{M}$ is the maximum estimated delay to get stable behavior of the controlled plant, as a result of the stability analysis (Section 3).
} 
where $K \in \Re^{m \times n}$ is a static state feedback control law such that the controlled system is stable and fulfils some design requirements. Thus, $A-B K$ is a Hurwitz matrix.

But if there is an output measurement time-varying delay, that is, for

$$
u_{k}=-K \tilde{x}_{k}=-K x_{k-d_{k}}
$$

the stability of the closed-loop is not guaranteed.

The robust stability of the closed-loop system (3)-(5) has been studied and reported in the cited literature: given the control law in (4), the time delay interval $\left\{d_{m}, d_{M}\right\}$ ensuring the system stability can be computed.

\subsection{Discrete time model based predictor}

To counteract the delay, a predicted state $\bar{x}$ (see Figure 1) will be used to provide the control input and its influence in the closed-loop robustness will be analyzed.

A measurement delay $h \in Z^{+}$is assumed to compute the predicted state (usually $h \in\left\{d_{m}, d_{M}\right\}$ ), being a predictor design parameter. The proposed predictor-based control scheme is

$$
u_{k}=-K \bar{x}_{k+h}
$$

where $\bar{x}_{k+h}$ is an $h$-step ahead prediction, that is

$$
\begin{aligned}
\bar{x}_{k+h} & =A^{h} x_{k-d_{k}}+\sum_{i=0}^{h-1} A^{h-i-1} B u_{k+i-h} \\
& =A^{h} x_{k-d_{k}}+\Sigma_{h, k}
\end{aligned}
$$

where $x_{k-d_{k}}$ is the current state measurement. The actual delay $d_{k}$ is randomly varying and unknown (Assumption A.2).

\subsection{Closed-loop behavior}

Concerning the dynamics of the closed-loop system, the following Lemma can be stated. 
Lemma 1 The predictor-based closed-loop controlled system composed of (3), (6) and (7), leads to

$$
x_{k+1}=(A-B K) x_{k}-B K A^{h} x_{k-d_{k}}+B K A^{h} x_{k-h}
$$

Proof: Combining (6) and (3), and taking into account (7), it yields

$$
x_{k+1}=A x_{k}-B K A^{h} x_{k-d_{k}}-B K \Sigma_{h, k}
$$

where $\Sigma_{h, k}$ is as defined in (7).

On the other hand, by recursively applying (3) $h$ steps, it results

$$
x_{k}=A^{h} x_{k-h}+\Sigma_{h, k}
$$

But $\Sigma_{h, k}$ can be eliminated between (10) and (9), leading to (8). This completes the proof.

\section{Delay-dependent stability condition for time-varying delay sys- tems}

In this section, a new stability condition for the predictor-based controlled system (8), where the control is computed by using the assumed measurement delay $h$ different from the actual one $d_{k}$ which is time-varying but bounded, is proposed.

The stability condition is based on a set of LMIs (Boyd et al. (1994)). The solution of the LMI problem, if a feasible one exists, can be easily found by any standard available software such as Matlab's LMI Control Toolbox, Gahinet et al. (1995). For a given delay lower bound $d_{m}$, the goal is to find the largest delay interval, $\delta=d_{M}-d_{m}$, ensuring the closed-loop stability.

To simplify the notation, the new matrices $A_{0}=(A-B K)$ and $A_{1}=$ $B K A^{h}$ are defined. Hence, from (8) it yields

$$
x_{k+1}=A_{0} x_{k}+A_{1} x_{k-h}-A_{1} x_{k-d_{k}}
$$

Theorem 1 System (11) is asymptotically stable if there exist matrices $P, Z_{1}, Z_{2}>$ $0 ; Q, Q_{m}, Q_{M}, Q_{h}, Z_{M} \geq 0$ and matrices $S_{1}, S_{2}, T_{1}, T_{2}$ satisfying the following matrix inequalities

$$
\left(\begin{array}{cc}
\Gamma & \delta S \\
\delta S^{T} & -\delta Z_{M}
\end{array}\right)<0 \quad\left(\begin{array}{cc}
\Gamma & \delta T \\
\delta T^{T} & -\delta Z_{M}
\end{array}\right)<0
$$


where

$$
\begin{aligned}
& S=\left(\begin{array}{lllllll}
0 & S_{1}^{T} & S_{2}^{T} & 0 & 0 & 0 & 0
\end{array}\right)^{T} \\
& T=\left(\begin{array}{lllllll}
0 & T_{1}^{T} & 0 & T_{2}^{T} & 0 & 0 & 0
\end{array}\right)^{T} .
\end{aligned}
$$

The symmetric matrix $\Gamma$ is defined as follows ${ }^{4}$ :

$$
\begin{aligned}
& \Gamma=\left(\begin{array}{ccccccc}
\Gamma_{1} & 0 & \Gamma_{2} & 0 & \Gamma_{3} & \Gamma_{4} & \Gamma_{5} \\
(*) & \Gamma_{6} & \Gamma_{7} & \Gamma_{8} & 0 & \Gamma_{9} & \Gamma_{10} \\
(*) & (*) & \Gamma_{11} & 0 & Z_{1} & 0 & 0 \\
(*) & (*) & (*) & \Gamma_{12} & 0 & 0 & 0 \\
(*) & (*) & (*) & (*) & \Gamma_{13} & \Gamma_{14} & \Gamma_{15} \\
(*) & (*) & (*) & (*) & (*) & -P & 0 \\
(*) & (*) & (*) & (*) & (*) & (*) & -Z
\end{array}\right) \text { where } \\
& \Gamma_{1}=-P+(\delta+1) Q+Q_{M}+Q_{m}+Q_{h}-Z_{2} \\
& \Gamma_{2}= \begin{cases}0 & h \leq d_{m} \\
Z_{2} & h>d_{m}\end{cases} \\
& \Gamma_{3}= \begin{cases}Z_{2} & h \leq d_{m} \\
0 & h>d_{m}\end{cases} \\
& \Gamma_{4}=A_{0}^{T} P \\
& \Gamma_{5}=\left(A_{0}-I\right)^{T} Z \\
& \Gamma_{6}=-Q+T_{1}+T_{1}^{T}-S_{1}-S_{1}^{T} \\
& \Gamma_{7}=S_{1}-S_{2}^{T} \\
& \Gamma_{8}=-T_{1}+T_{2}^{T} \\
& \Gamma_{9}=-A_{1}^{T} P \\
& \Gamma_{10}=-A_{1}^{T} Z \\
& \Gamma_{11}=-Q_{m}+S_{2}+S_{2}^{T}-Z_{1}+ \begin{cases}0 & h \leq d_{m} \\
-Z_{2} & h>d_{m}\end{cases} \\
& \Gamma_{12}=-Q_{M}-T_{2}-T_{2}^{T} \\
& \Gamma_{13}=-Q_{h}-Z_{1}+ \begin{cases}-Z_{2} & h \leq d_{m} \\
0 & h>d_{m}\end{cases} \\
& \Gamma_{14}=A_{1}^{T} P \\
& \Gamma_{15}=A_{1}^{T} Z \\
& Z=\rho_{1}^{2} Z_{2}+\left(d_{m}-h\right)^{2} Z_{1}+\delta Z_{M} \\
& \rho_{1}=\min \left(d_{m}, h\right)
\end{aligned}
$$

${ }^{4} I(0)$ denotes the identity (zero) matrix of appropriate dimension and $(*)$ represents a term induced by symmetry. 
Note that $\delta=d_{M}-d_{m}$ is an analysis parameter.

Proof: Following the ideas in Guo and Li (2009) and Garcia et al. (2010), a Lyapunov-Krasovskii function candidate is defined as

$$
V(k)=V_{1}(k)+V_{2}(k)+V_{3}(k)+V_{4}(k)+V_{5}(k)
$$

where

$$
\begin{aligned}
V_{1}(k) & =x_{k}^{T} P x_{k} \\
V_{2}(k) & =\sum_{i=k-d_{k}}^{k-1} x_{i}^{T} Q x_{i} \\
V_{3}(k) & =\sum_{i=k-d_{m}}^{k-1} x_{i}^{T} Q_{m} x_{i}+\sum_{i=k-d_{M}}^{k-1} x_{i}^{T} Q_{M} x_{i}+\sum_{i=k-h}^{k-1} x_{i}^{T} Q_{h} x_{i} \\
V_{4}(k) & =\sum_{j=-d_{M}+1}^{-d_{m}} \sum_{i=k+j}^{k-1} x_{i}^{T} Q x_{i} \\
V_{5}(k) & =\rho_{1} \sum_{i=-\rho_{1}}^{-1} \sum_{m=k+i}^{k-1} \nu_{m}^{T} Z_{2} \nu_{m}+\left|d_{m}-h\right| \sum_{i=-\rho_{2}}^{-\rho_{1}-1} \sum_{m=k+i}^{k-1} \nu_{m}^{T} Z_{1} \nu_{m}+ \\
& +\sum_{i=-d_{M}}^{-d_{m}-1} \sum_{m=k+i}^{k-1} \nu_{m}^{T} Z_{M} \nu_{m}
\end{aligned}
$$

with $\nu_{k}=x_{k+1}-x_{k}, \rho_{1} \doteq \min \left(d_{m}, h\right)$ and $\rho_{2} \doteq \max \left(d_{m}, h\right)$.

Then, the closed-loop system (11) will be asymptotically stable if the forward difference $\Delta V(k)=V(k+1)-V(k)$ satisfies

$$
\Delta V(k)=\Delta V_{1}(k)+\Delta V_{2}(k)+\Delta V_{3}(k)+\Delta V_{4}(k)+\Delta V_{5}(k)<0
$$

After some lengthy algebraic manipulations (see appendix), it yields

$$
\Gamma+\left(d_{k}-d_{m}\right) S Z_{M}^{-1} S^{T}+\left(d_{M}-d_{k}\right) T Z_{M}^{-1} T^{T}<0
$$

Note that the first term in the above inequality is linear affine function in the variable $d_{k}$. Taking into account the same considerations as Guo and $\mathrm{Li}$ (2009) the equivalence between this inequality and the following LMI constraints can be proved 


$$
\begin{aligned}
& \Gamma+\delta S Z_{M}^{-1} S^{T}<0 \\
& \Gamma+\delta T Z_{M}^{-1} T^{T}<0
\end{aligned}
$$

Finally, applying Schur complement to the above inequalities result on the LMI constraints (12). This completes the proof.

This theorem allows to check the improvement of using the state predictor in the feedback control. The following numerical examples, already reported in the literature, illustrate this advantage.

\section{Numerical examples}

The stability of a state delayed plant defined by

$$
x_{k+1}=A x_{k}+A_{d} x_{k-d_{k}}
$$

where $d_{k}$ fulfils Assumption A.2, has been widely studied in the literature (see for example Gao and Chen (2007); Guo and Li (2009); Meng et al. (2010)).

This system is equivalent to (3) with delayed state feedback (5), leading to the equivalence

$$
A_{d}=-B K
$$

Thus, the results achieved by using the proposed predictor are compared with those obtained by the above referenced approaches where a delayed state feedback control is used.

Example 1 Consider the open-loop unstable model of an inverted pendulum (see Example 3 in Gao and Chen (2007)). In that paper, a sampling period of $30 \mathrm{~ms}$ was adopted. Here, a shorter sampling period is used ( $T=10$ $m s$ ), in order to better show the influence of the delay (which is expressed as multiples of T). The ZOH-discretized model of the inverted pendulum is

$$
x_{k+1}=\left(\begin{array}{ll}
1.0009 & 0.0100 \\
0.1730 & 1.0009
\end{array}\right) x_{k}+10^{-3}\left(\begin{array}{l}
-0.0088 \\
-1.7652
\end{array}\right) u_{k}
$$

The poles of the open-loop unstable system are $\{1.0425,0.9593\}$. Following Gao and Chen (2007) a stabilizing state-feedback control gain $K$ was computed

$$
K=-\left[\begin{array}{ll}
102.9100 & 80.7916
\end{array}\right]
$$


assigning the poles of the delay-free closed-loop system $(A-B K)$ at $\{0.9994,0.8588\}$.

Now, the existence of a time varying $d_{k}$ measurement delay is assumed (see Figure 1). Two options are considered:

1. The feedback control input is the delayed state (5), $u_{k}=-K x_{k-d_{k}}$.

2. The predicted state (7) is used when computing the control (6), $u_{k}=$ $-K \bar{x}_{k+h}$.

Given $d_{m}$, the goal is to obtain the upper value of $d_{M}$ such that the closed-loop system remains stable. In Table, 1 comparative results using Theorem 1 in Guo and Li (2009), Theorem 1 and 3 in Gao and Chen (2007), Theorem 1 in Meng et al. (2010) $)^{5}$, without the predictor, as well as the results of the proposed stability condition in Theorem 1, when the predictor is used, are summarized.

Table 1: Calculation of $d_{M}$ given $d_{m}$

\begin{tabular}{c|ccccc}
\hline \hline state feedback & & \multicolumn{5}{c}{$d_{m}$} \\
\hline & & 1 & 4 & 7 & 10 \\
\hline delayed & Th.1 Guo(2009) & 8 & 8 & 8 & infeasible \\
\hline delayed & Th.1 Gao(2007) & 8 & 8 & 8 & infeasible \\
\hline delayed & Th.3 Gao(2007) & 8 & 8 & 9 & infeasible \\
\hline delayed & Th.1 Meng & 8 & 8 & 9 & 10 \\
\hline predicted & Th.1 $\left(h=d_{m}\right)$ & 8 & 10 & 13 & 15 \\
\hline
\end{tabular}

The proposed approach has been applied assuming $h=d_{m}$. As shown, the use of the predictor enlarges the stability interval. For a minimum delay $d_{m}=10$ it is not possible to prove the time-varying delay-dependent stability of the delayed state feedback controlled plant whereas it is ensured until a maximum delay of $d_{M}=15$ if the predictor (7) is used in the feedback control.

Example 2 Now, consider the following double integrator plant (which is a simple model of the experimental platform used afterwards)

$$
\dot{x}(t)=\left(\begin{array}{ll}
0 & 1 \\
0 & 0
\end{array}\right) x(t)+\left(\begin{array}{c}
0 \\
0.1050
\end{array}\right) u(t)
$$

\footnotetext{
${ }^{5}$ Where the less conservative delay-partitioning approach has been used.
} 
with a sampling period of $T=10 \mathrm{~ms}$, the following control law $u_{k}=-[109.9907$ $50.0020] x_{k}$ stabilizes the plant assigning the poles of the delay-free closed-loop system at $\{0.9735 \pm 0.0212 i\}$. Then, following the same procedure as in the previous example, the results in Table 2 are obtained.

Table 2: Calculation of $d_{M}$ given $d_{m}$

\begin{tabular}{c|ccccc}
\hline \hline state feedback & \multicolumn{5}{c}{$d_{m}$} \\
\hline & & 5 & 10 & 20 & 30 \\
\hline delayed & Th.1 Guo(2009) & 19 & 19 & infeasible & infeasible \\
\hline delayed & Th.1 Gao(2007) & 19 & 19 & infeasible & infeasible \\
\hline delayed & Th.3 Gao(2007) & 19 & 19 & infeasible & infeasible \\
\hline delayed & Th.1 Meng & 19 & 19 & 20 & infeasible \\
\hline predicted & Th.1 $\left(h=d_{m}\right)$ & 21 & 25 & 32 & 41 \\
\hline
\end{tabular}

Also in this case, better time-varying measurement delay robustness is achieved by using the state predictor in the feedback control. Note that, when $d_{m}=30$ is not possible to prove the stability of the delayed state feedback controlled plant whereas it is ensured until a maximum delay of $d_{M}=41$ if the predictor (7) is used in the feedback control.

Remark 1 The main objective of this work is to prove that the proposed predictor-controller scheme improves robustness against uncertainties in the time-varying measurement delay. Theorem 1 provides a conservative bound (which could be improved by choosing another $h$ ) in the robust stability. This is a matter of current research.

\section{Robust-stability of the closed-loop system}

In this section, robustness of the designed control scheme to variations in all the parameters of the system model are analyzed.

Removing the assumptions A.1, A.2, and A.3 and following the ideas in Lozano et al. (2004); Cloosterman et al. (2010), the system (1) can be modeled in discrete-time as:

$$
x_{k+1}=A x_{k}+B u_{k}+\Delta f_{k}
$$


where $f_{k}=\left[x_{k}^{T}, u_{k}^{T}, u_{k-1}^{T}\right]^{T}$ is the vector of system variables ${ }^{6}$ and the uncertainty matrix $\Delta$ is defined as:

$$
\Delta=\left(\begin{array}{lll}
\Delta_{A} & \Delta_{B} & \Delta_{B 1}
\end{array}\right)=\gamma G \Delta_{k}\left(\begin{array}{lll}
H_{A} & H_{B} & H_{B 1}
\end{array}\right)
$$

where $G, H_{A}, H_{B}, H_{B 1}$ are some constant matrices of appropriate dimensions, $\Delta_{k}$ is a real uncertain matrix function with Lebesgue measurable elements satisfying $\Delta_{k}^{T} \Delta_{k} \leq I$, and the scalar $\gamma>0$ determines the size of the uncertainties.

Define an augmented state representation of the system (21) together with the control law (6) and the predictor (7). The autonomous system can be represented as $^{7}$

$$
\bar{x}_{k+1}=\bar{A}_{c l}\left(d_{i}\right) \bar{x}_{k}
$$

where

$$
\begin{aligned}
& \bar{x}_{k}=\left(\begin{array}{llll}
x_{k}^{T} \cdots & x_{k-\bar{d}}^{T} & u_{k-1}^{T} \cdots & u_{k-h}^{T}
\end{array}\right)^{T} \\
& \bar{d}=\max \left(d_{M}, h\right)
\end{aligned}
$$

and the state matrix $\bar{A}_{c l}\left(d_{i}\right)$, assuming an actual delay $d_{k}=d_{i}$, is

$$
\begin{gathered}
\bar{A}_{c l}\left(d_{i}\right)=\bar{A}\left(d_{i}\right)+\gamma \bar{G} \Delta_{k} \bar{H}\left(d_{i}\right) \\
\bar{A}\left(d_{i}\right)=\left(\begin{array}{ccccc}
\bar{A}_{11}\left(d_{i}\right) & \bar{A}_{12} \\
\bar{A}_{21}\left(d_{i}\right) & \bar{A}_{22}
\end{array}\right) \\
\bar{A}_{11}\left(d_{i}\right)=\left(\begin{array}{ccccc}
A+B K_{0}\left(d_{i}\right) & B K_{1}\left(d_{i}\right) & \cdots & \cdots & B K_{\bar{d}}\left(d_{i}\right) \\
I & 0 & \cdots & \cdots & 0 \\
0 & I & \cdots & \cdots & 0 \\
\cdots & \cdots & \cdots & \cdots & \cdots \\
0 & 0 & \cdots & I & 0
\end{array}\right)
\end{gathered}
$$

\footnotetext{
${ }^{6}$ Note that the previous input, $u_{k-1}$, is included in $f_{k}$, as some variations in the sampling period, (Assumption A.1), may force this dependence.

${ }^{7}$ Note that the closed-loop realization also involves all the past state and system input delayed variables.
} 


$$
\begin{aligned}
& \bar{A}_{12}=\left(\begin{array}{ccc}
-B K B & \cdots & -B K A^{h-1} B \\
0 & \cdots & 0 \\
\cdots & \cdots & \cdots \\
0 & \cdots & 0
\end{array}\right) \\
& \bar{A}_{21}\left(d_{i}\right)=\left(\begin{array}{cccc}
K_{0}\left(d_{i}\right) & K_{1}\left(d_{i}\right) & \cdots & K_{\bar{d}}\left(d_{i}\right) \\
0 & 0 & \cdots & 0 \\
\cdots & \cdots & \cdots & \cdots \\
0 & 0 & \cdots & 0
\end{array}\right) \\
& \bar{A}_{22}=\left(\begin{array}{cccc}
-K B & \cdots & -K A^{h-2} B & -K A^{h-1} B \\
I & \cdots & \cdots & 0 \\
0 & \cdots & \cdots & 0 \\
\cdots & \cdots & \cdots & \cdots \\
0 & \cdots & I & 0
\end{array}\right) \\
& \bar{G}=\left(\begin{array}{llll}
G & 0 & \cdots & 0
\end{array}\right)^{T} \\
& \bar{H}\left(d_{i}\right)=\left(\begin{array}{ll}
\bar{H}_{1}\left(d_{i}\right) & \bar{H}_{2}
\end{array}\right) \\
& \bar{H}_{1}\left(d_{i}\right)=\left(\begin{array}{llll}
H_{A}+H_{B} K_{0}\left(d_{i}\right) & H_{B} K_{1}\left(d_{i}\right) & \cdots & H_{B} K_{\bar{d}}\left(d_{i}\right)
\end{array}\right) \\
& \bar{H}_{2}=\left(\begin{array}{llll}
-H_{B} K B+H_{B 1} & -H_{B} K A B & \cdots & -H_{B} K A^{h-1} B
\end{array}\right) \\
& K_{j}\left(d_{i}\right)= \begin{cases}-K A^{h} & j=d_{i} \\
0 & j \neq d_{i}\end{cases}
\end{aligned}
$$

By applying a similar procedure as in Gonzalez et al. (2011), the following Theorem is obtained:

Theorem 2 The closed-loop system (21) with predictor-based scheme (6) is robustly stable if there exists $\bar{L}>0$ such that the following LMI's hold for every $d_{i}=d_{m}, d_{m}+1, \cdots, d_{M}$

$$
\left(\begin{array}{ccc}
-\bar{L}+\bar{H}\left(d_{i}\right)^{T} \bar{H}\left(d_{i}\right) & \bar{A}\left(d_{i}\right)^{T} \bar{L} & 0 \\
(*) & -\bar{L} & \bar{L} \bar{G} \\
(*) & (*) & -\gamma^{-2} I
\end{array}\right)<0
$$

Moreover, the maximum tolerance to uncertainties keeping stability can be obtained by solving the following convex optimization problem: $\min \gamma^{-2}$ subject to (25). 
Proof: Propose the complete quadratic Lyapunov functional candidate $V(k)=\bar{x}_{k}^{T} \bar{P} \bar{x}_{k}, \bar{P}>0$ where the augmented state is defined in Equation (23). The closed-loop stability can be proved if the following LMI constraints hold for every $d_{i}=d_{m}, d_{m}+1, \cdots, d_{M}$.

$$
-\bar{P}+\bar{A}_{c l}\left(d_{i}\right)^{T} \bar{P} \bar{A}_{c l}\left(d_{i}\right)<0
$$

Applying Schur Complement, the following is obtained

$$
\left(\begin{array}{cc}
-\bar{P} & \bar{A}_{c l}\left(d_{i}\right)^{T} \bar{P} \\
(*) & -\bar{P}
\end{array}\right)<0
$$

That can be put in the following form, taking into account that $\bar{A}_{c l}\left(d_{i}\right)=$ $\bar{A}\left(d_{i}\right)+\gamma \bar{G} \Delta_{k} \bar{H}\left(d_{i}\right)$

$$
\begin{array}{r}
\left(\begin{array}{cc}
-\bar{P} & \bar{A}\left(d_{i}\right)^{T} \bar{P} \\
(*) & -\bar{P}
\end{array}\right)+\gamma\left(\begin{array}{c}
0 \\
P \bar{G}
\end{array}\right) \Delta_{k}\left(\begin{array}{ll}
\bar{H}\left(d_{i}\right) & 0
\end{array}\right)+ \\
\gamma\left(\begin{array}{ll}
\bar{H}\left(d_{i}\right) & 0
\end{array}\right)^{T} \Delta_{k}^{T}\left(\begin{array}{c}
0 \\
P \bar{G}
\end{array}\right)^{T}<0
\end{array}
$$
that

The previous inequality is satisfied for some scalar $\epsilon>0$, Xie (1996), such

$$
\begin{aligned}
& \left(\begin{array}{cc}
-\bar{P} & \bar{A}\left(d_{i}\right)^{T} \bar{P} \\
(*) & -\bar{P}
\end{array}\right)+\epsilon \gamma^{2}\left(\begin{array}{c}
0 \\
P \bar{G}
\end{array}\right)\left(\begin{array}{ll}
0 & \bar{G}^{T} P
\end{array}\right)+ \\
& \epsilon^{-1}\left(\begin{array}{c}
\bar{H}\left(d_{i}\right)^{T} \\
0
\end{array}\right)\left(\begin{array}{ll}
\bar{H}\left(d_{i}\right) & 0
\end{array}\right)<0
\end{aligned}
$$

Applying again the Schur Complement

$$
\left(\begin{array}{ccc}
-\bar{P}+\epsilon^{-1} \bar{H}\left(d_{i}\right)^{T} \bar{H}\left(d_{i}\right) & \bar{A}\left(d_{i}\right)^{T} \bar{P} & 0 \\
(*) & -\bar{P} & \bar{P} \bar{G} \\
(*) & (*) & -\gamma^{-2} \epsilon^{-1} I
\end{array}\right)<0
$$

and multiplying by $\epsilon$

$$
\left(\begin{array}{ccc}
-\epsilon \bar{P}+\bar{H}\left(d_{i}\right)^{T} \bar{H}\left(d_{i}\right) & \epsilon \bar{A}\left(d_{i}\right)^{T} \bar{P} & 0 \\
(*) & -\epsilon \bar{P} & \epsilon \bar{P} \bar{G} \\
(*) & (*) & -\gamma^{-2} I
\end{array}\right)<0
$$


Finally, by defining $\bar{L}=\epsilon \bar{P}$ the expression (25) is obtained. This completes the proof.

Example 3 Consider the process in Example 2 with uncertainties as described in Equation (21) with $G=10^{-4}\left(\begin{array}{ll}1 & 1\end{array}\right)^{T}, H_{A}=\left(\begin{array}{ll}1 & 1\end{array}\right)$ and $H_{B}=1$.

Assuming that both bound are fixed, $\delta=d_{M}-d_{m}$, the objective is to find the upper value of $\gamma$ such that the closed-loop system remains stable. By using Theorem 2, for $h=d_{m}$, the maximum $\gamma$ is shown for some delay intervals in Table 3.

Table 3: Allowable upper bound $\bar{\gamma}$ for some delay intervals $\left(h=d_{m}\right)$

\begin{tabular}{ccccccc}
\hline$d_{m}$ & 3 & 6 & 9 & 12 & 15 & 18 \\
\hline \hline $\bar{\gamma}(\delta=2)$ & 1.0440 & 1.0148 & 0.9867 & 0.9591 & 0.9291 & 0.8882 \\
\hline $\bar{\gamma}(\delta=3)$ & 1.0080 & 0.9751 & 0.9409 & 0.9062 & 0.8473 & 0.8296 \\
\hline $\bar{\gamma}(\delta=4)$ & 0.9712 & 0.9308 & 0.8904 & 0.8446 & 0.7802 & 0.7336 \\
\hline $\bar{\gamma}(\delta=5)$ & 0.9290 & 0.8797 & 0.8297 & 0.7729 & 0.7031 & 0.6428 \\
\hline
\end{tabular}

Remark 2 Note that, if another measurement delay $h$ is considered (for example, $h=d_{M}$ ), robustness against model uncertainties is improved (see Table 4). This result has been also verified by simulation. As previously mentioned, the optimal value of this parameter is a matter of current research.

Table 4: Allowable upper bound $\bar{\gamma}$ for some delay intervals $\left(h=d_{M}\right)$

\begin{tabular}{ccccccc}
\hline$d_{m}$ & 3 & 6 & 9 & 12 & 15 & 18 \\
\hline $\bar{\gamma}(\delta=2)$ & 1.0905 & 1.0702 & 1.0493 & 1.0302 & 1.0046 & 0.9837 \\
\hline $\bar{\gamma}(\delta=3)$ & 1.0826 & 1.0616 & 1.0385 & 1.0036 & 0.9632 & 0.9436 \\
\hline $\bar{\gamma}(\delta=4)$ & 1.0743 & 1.0505 & 1.0065 & 0.9807 & 0.9406 & 0.9267 \\
\hline $\bar{\gamma}(\delta=5)$ & 1.0615 & 1.0321 & 1.0009 & 0.9655 & 0.8774 & 0.8359 \\
\hline
\end{tabular}

Now, some simulation results are reported. Assume the initial condition to be $x_{k}=\left[\begin{array}{ll}1 & 1\end{array}\right]^{T}$ for $k \leq 0$. In addition, let the delay $d_{k}$ change randomly between $d_{m}=18$ and $d_{M}=23$ as depicted in Figure 2, and the variable process uncertainties being defined by $\gamma=0.8359$ and $\Delta_{k}$ changing randomly between -1 and 1 . The predictor-based control assumes $h=d_{M}=23$. Then, the state response of the double integrator is given in Figure 3. It can be seen that the system is asymptotically stable. 


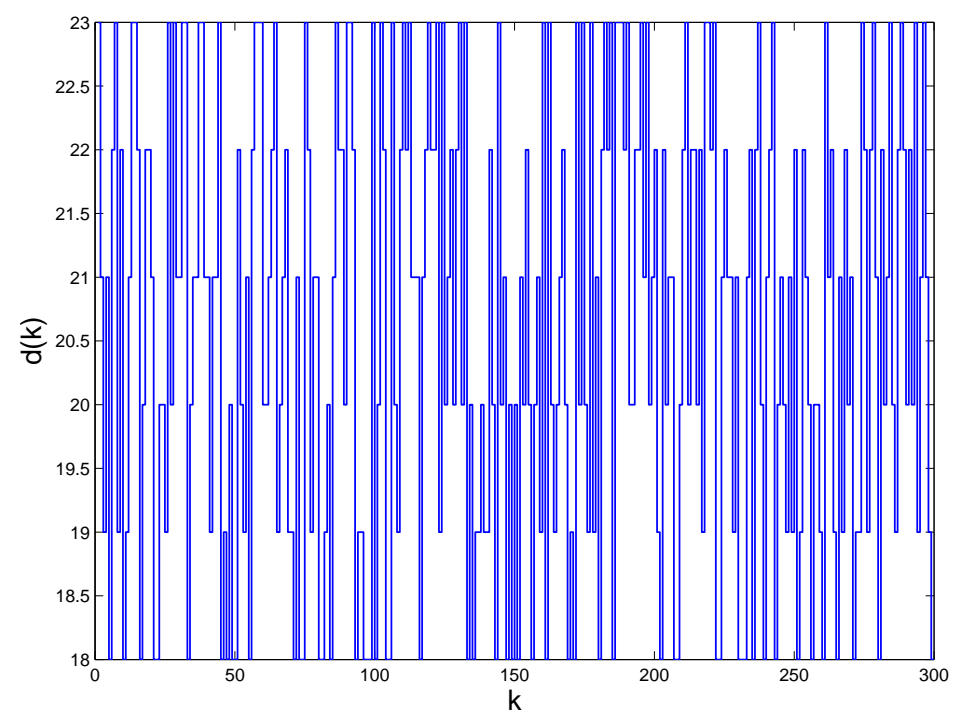

Figure 2: The time-varying delay in Example 3.

\section{Experimental results}

The goal of this section is to illustrate the effectiveness and robustness of the proposed predictor-based control scheme in a real-time closed-loop system. For that purpose, the real-time platform shown in Figure 4 has been used. The platform is composed of a ground control unit (GCU), developed using Linux RT, and a quad-rotor prototype equipped with a wireless Integrated Measurement Unit (IMU) to measure the angular position and velocity (yaw, pitch and roll angles).

Assuming the pitch and yaw angles are zero, the roll axis torque is controlled by the forces generated by two propellers, and the following simplified model can be obtained, Castillo et al. (2005):

$$
\ddot{\phi}=K_{\phi}\left(V_{r}-V_{l}\right)
$$

where $V_{r}, V_{l}$ are the right and left motor voltages respectively, $K_{\phi}=0.1050$ is a constant representing the inertia moment of the body and $\ddot{\phi}$ denotes the roll acceleration of the system.

Define $x=\left[\begin{array}{ll}\phi & \dot{\phi}\end{array}\right]^{T}$ as the state vector. Then, the roll angle dynamics can be written as

$$
\dot{x}(t)=A_{c} x(t)+B_{c} u(t)
$$




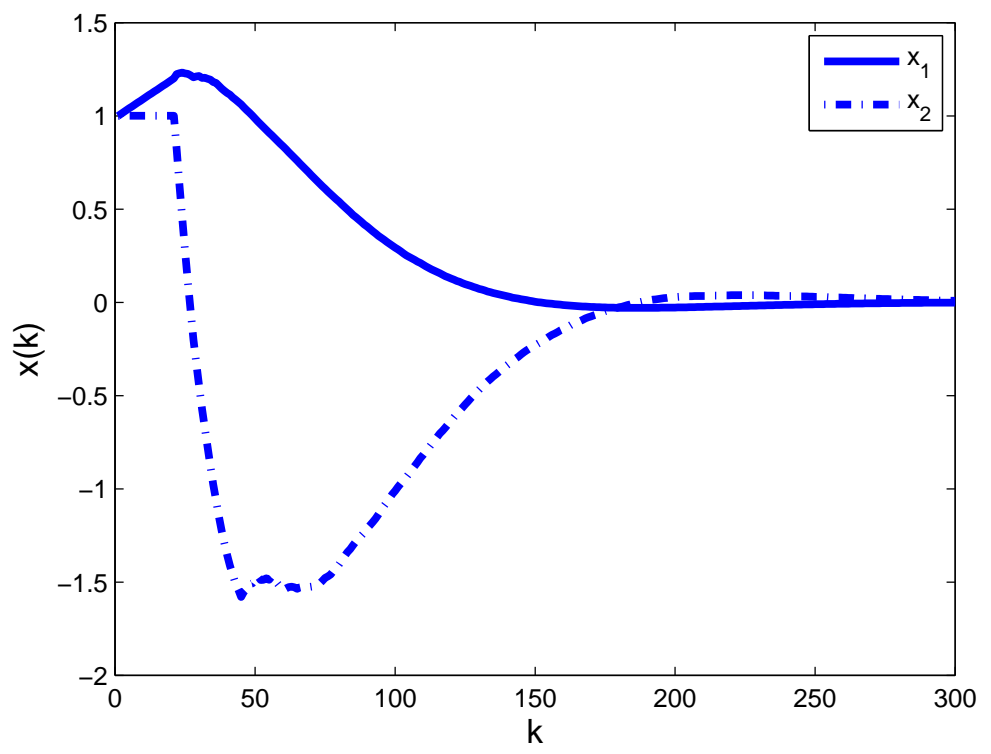

Figure 3: State evolution with predictor-based control $\left(h=d_{M}\right)$, timevarying delay $18 \leq d_{k} \leq 23$ and $\gamma=0.8359$.

with

$$
A_{c}=\left(\begin{array}{cc}
0 & 1 \\
0 & 0
\end{array}\right) ; \quad B_{c}=\left(\begin{array}{cc}
0 & 0 \\
K_{\phi} & -K_{\phi}
\end{array}\right)
$$

and

$$
u(t)=\left(\begin{array}{c}
V_{r} \\
V_{l}
\end{array}\right) .
$$

Note that this model has been already used in Example 2. Again, the sampling period for all experiments is assumed to be $T=10 \mathrm{~ms}$.

As before, a stabilizing control law as the one used in Example 2 is designed. Then ${ }^{8}$, the following control input is assumed

$$
u_{k}=\left(\begin{array}{cc}
-109.9907 & -50.0020 \\
109.9907 & 50.0020
\end{array}\right) x_{k}
$$

\footnotetext{
${ }^{8}$ Note that the purpose of this section is not to validate the performance of the control law, which is assumed to be given, but to illustrate the effectiveness of the proposed predictor-scheme with respect to random delays in a real-time application.
} 


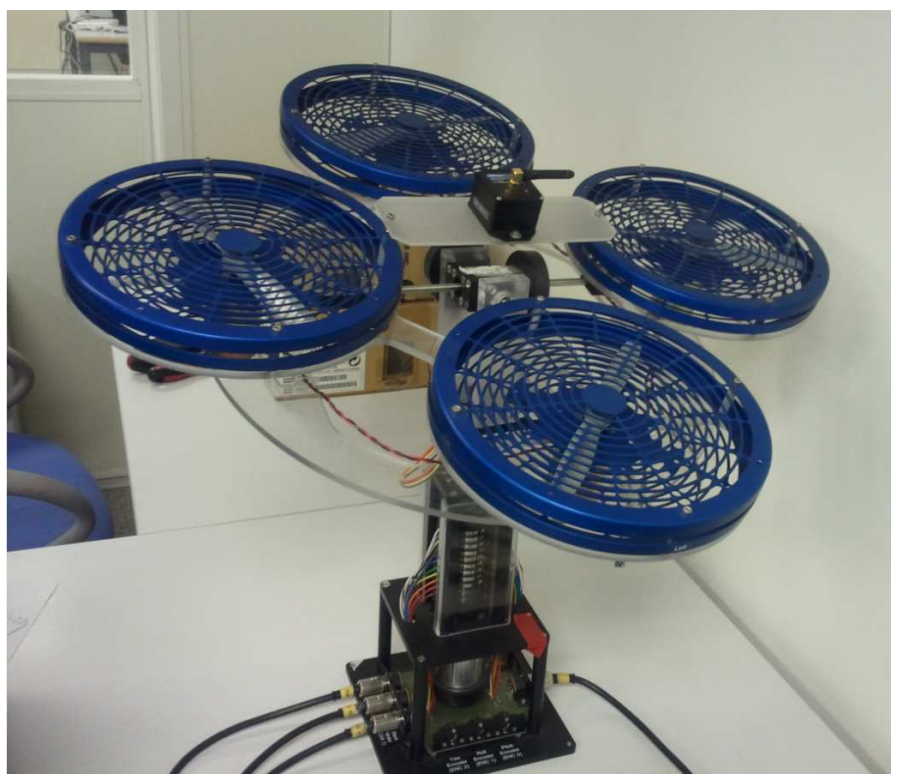

Figure 4: Quad-rotor system with a wireless Integrated Measurement Unit (IMU).

In order to test the predictor scheme, a square wave function, with $5^{\circ}$ of magnitude, is proposed as the desired reference position and the following experiments are run.

\section{First experiment:}

The first experiment is realized applying the controller (32) without any additional delay. Figure 5 illustrates the behavior of the system. Notice in this figure that the system remains stable with a constant error $e_{\phi}=1^{\circ}$.

\section{Second experiment:}

The second experiment is carried out adding a random time-varying delay in the measurement state. No prediction is implemented. That is, the state feedback measurement signal is delayed as $x_{k-d_{k}}$, where $d_{k}=d_{m}+\delta_{k}, d_{m}$ is the fixed minimum discrete delay, and $\delta_{k}$ is the random number of the delayed samples here considered ${ }^{9}\left(1 \leq \delta_{k} \leq 2\right)$. The output response when this delay is activated is shown in Figure 6 . For $t<8 \mathrm{~s}$ and $d_{k}=0$, the

\footnotetext{
${ }^{9}$ In practical applications this is a usual range of time delay variations. Nevertheless some other intervals can be considered.
} 


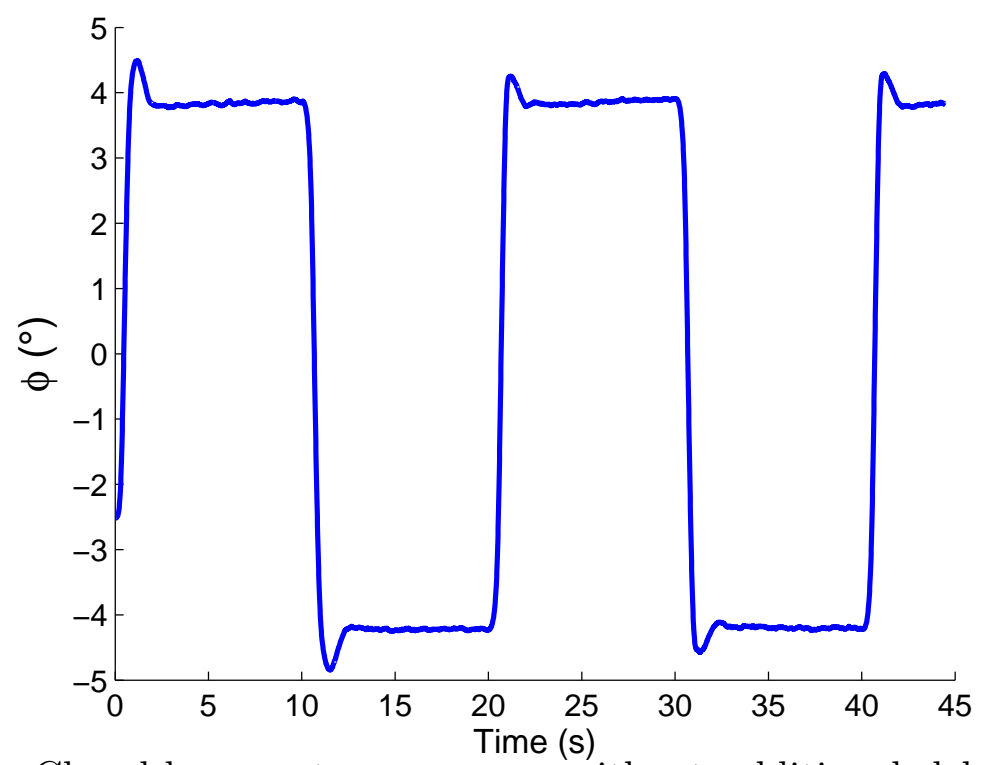

Figure 5: Closed-loop system response without additional delay (nominal case) using a square wave function as a desired angle.

response in Figure 5 is reproduced. The delay is increased to $3 \leq d_{k} \leq 5$ at $t=8 \mathrm{~s}$, the system remaining stable. If the additional delay is further increased, $5 \leq d_{k} \leq 7$ at $t \geq 18 \mathrm{~s}$, the system becomes unstable (see Figure $6)$.

The improvement achieved by using the predictor scheme as well as its robustness in presence of bigger delays is illustrated in the next experiment.

\section{Third experiment:}

In this experiment, the proposed predictor control scheme is implemented with the previous control law (32), and $h=8$. Similar to the previous experiment, the measurement state is delayed as depicted in Figure 8, where $d_{k} \in\{5,11\}$ and $\left|d_{k+1}-d_{k}\right| \leq 2$. Note that under the same conditions as the previous experiment $(t<25 \mathrm{~s})$, the closed-loop system remains stable. Moreover, the behavior obtained using the proposed scheme is very similar to the delay-free case (see Figures 5 and 7 ).

In order to prove the robustness of the predictor-based control scheme, the additional delay is increased. At $t=25 \mathrm{~s}$, the lower bound delay is set at $d_{m}=7$, the system behavior is not much affected. But, for longer delays, $d_{m}=9$ at time $t=43\left(d_{k} \in\{9,11\}\right)$, the transient response degrades (see Figure 7). 


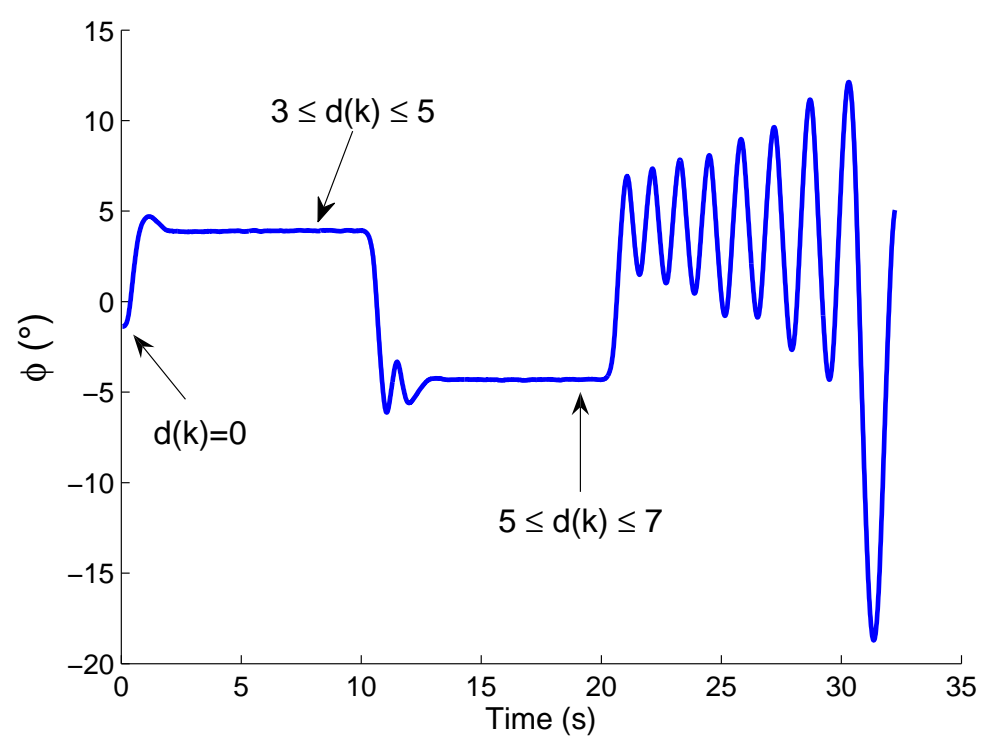

Figure 6: Closed-loop system response without predictor.

This result illustrates the use of the predictor scheme to restore the nominal closed-loop behavior, even when the time-varying delay-range is subject to some uncertainties.

\section{Conclusions}

In this paper a DT predictor-based control scheme for time-varying measurementdelay systems has been analyzed. The prediction horizon $h(7)$, is a design parameter, its optimal value being determined by de plant model and the time delays bound. Currently this is a matter of research. This control has been shown to be robust with respect to uncertain and time-varying bounded delays, besides the possible parametric model uncertainties, coping with long delays. Furthermore, the controlled plant performance remains similar to that of the nominal plant.

Other than the theoretical result providing a set of LMI constraints to analyze the stability of a predictor-based control applied to a time varying delay unstable plant, uncertainties in the knowledge of plant-model and sampling pattern parameters are also considered. The resulting closed-loop system is shown to be robust with respect to these uncertainties. 


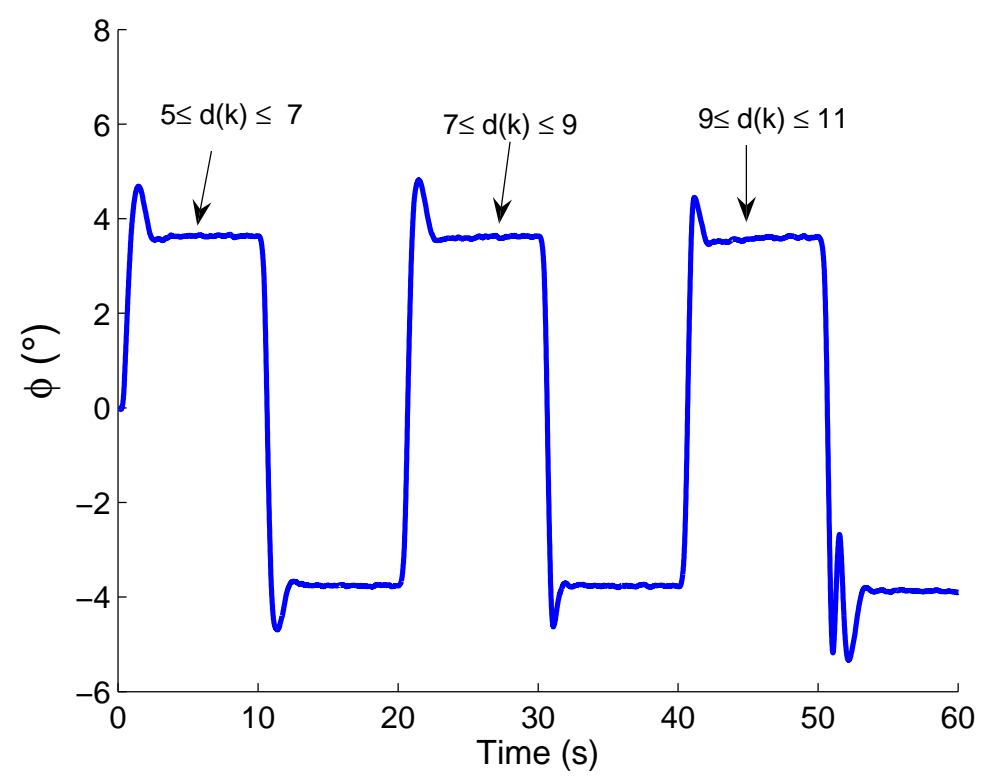

Figure 7: Closed-loop system response with predictor and additional timevarying delay.

The delay interval $\delta=d_{M}-d_{m}$ is an analysis parameter allowing to determine an interval providing stability in which the prediction horizon $h \in\left\{d_{M}, d_{m}\right\}$ should be selected.

One of the main contributions of this work concerns to the digital implementation and the experimental validation of the proposed algorithm by stabilizing the roll angle of a quad-rotor helicopter. Real-time experiments have enlightened the performance of the prediction based controller and have satisfactorily demonstrated its robustness with respect to inherent plant model uncertainties and additional delay measurement errors. Note that, this prototype is unstable with very fast dynamics where losing the information of one sampling period could be critical, and an unknown delay introduced by the wireless IMU is always present.

As already mentioned, the formal analysis of the influence of the predictor design parameter $(h)$ is a matter of current research. 


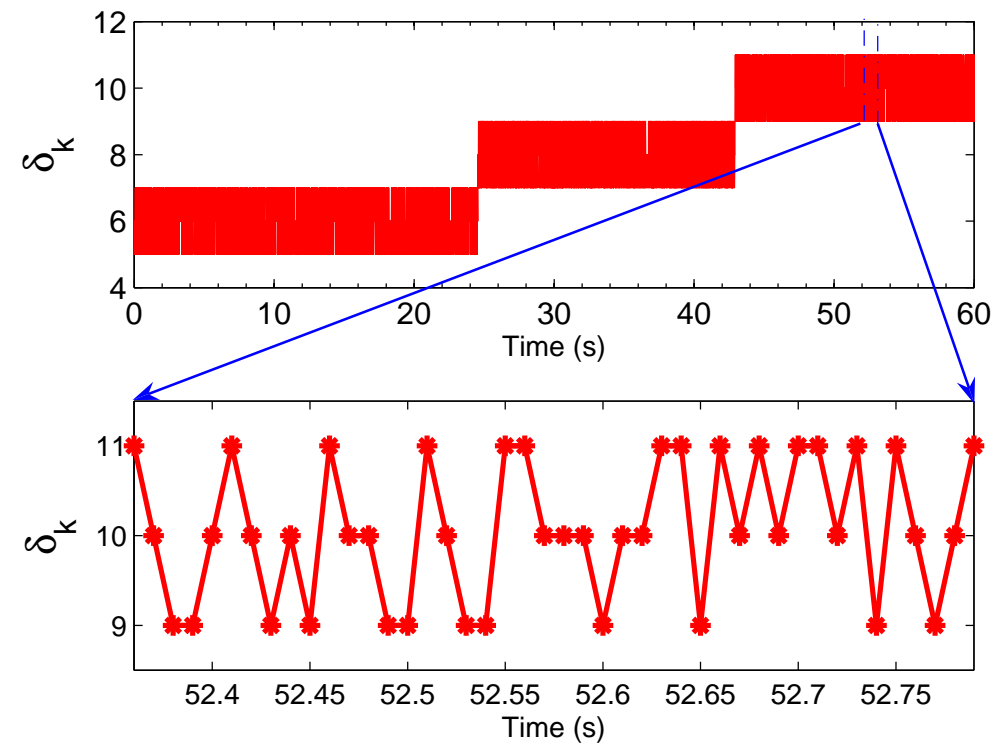

Figure 8: Additional random time-varying delays applied in the system.

\section{Acknowledgments}

The authors thank the Associate Editor and anonymous reviewers for their valuable comments and suggestions.

\section{References}

Astrom, K. J., Wittenmark, B., 1997. Computer-Controlled Systems - Theory and Design. Pretince-Hall, third edition.

Boukas, E.-K., 2006. Discrete-time systems with time-varying time delay:stability and stabilization. Mathematical Problems in Engineering 2006, 1-10.

Boyd, S., El Ghaoui, L., Feron, E., Balakrishnan, V., 1994. Linear matrix inequalities in system and control theory. Vol. 15. Society for Industrial and Applied Mathematics, Philadelphia.

Castillo, P., Lozano, R., Dzul, A., 2005. Modelling and control of mini flying machines. Springer-Verlag. 
Cloosterman, M., Hetel, L., van de Wouw, N., Heemels, W., Daafouz, J., Nijmeijer, H., 2010. Controller synthesis for networked control systems. Automatica.

Du, D., Jiang, B., Zhou, S., 2008. Delay-dependent robust stabilisation of uncertain discrete-time switched systems with time-varying state delay. International Journal of Systems Science 39 (3), 305-313.

Gahinet, P., Nemirovskii, A., Laub, A., Chilali, M., 1995. The lmi control toolbox. In: Decision and Control, 1994., Proceedings of the 33rd IEEE Conference on. Vol. 3. IEEE, pp. 2038-2041.

Gao, H., Chen, T., 2007. New results on stability of discrete-time systems with time-varying state delay. IEEE Transactions on Automatic Control $52(2), 328-334$.

Garcia, P., Castillo, P., Lozano, R., Albertos, P., 2006. Robustness with respect to delay uncertainties of a predictor-observer based discrete-time controller. In: Decision and Control, 2006 45th IEEE Conference on. IEEE, pp. 199-204.

Garcia, P., Gonzalez, A., Castillo, P., Lozano, R., Albertos, P., 2010. Robustness of a discrete-time predictor-based controller for time-varying measurement delay. International Federation of Automatic Control (http://tds2010.fs.cvut.cz).

Gonzalez, A., Sala, A., Garcia, P., Albertos, P., 2011. Robustness analysis of discrete predictor-based controllers for input-delay systems. International Journal of Systems Science (DOI:10.1080/00207721.2011.600469).

Guangdeng, Z., Linlin, H., Hongyong, Y., 2009. Further results concerning delay-dependent $\mathrm{h}_{\infty}$ control for uncertain discrete-time systems with timevarying delay. Mathematical Problems in Engineering 2009.

Guo, Y., Li, S., 2009. New stability criterion for discrete-time systems with interval time-varying state delay. In: Proceedings of the 48th IEEE Conference on Decision and Control and the 2009 28th Chinese Control Conference. CDC/CCC 2009. IEEE, pp. 1342-1347. 
Guzman, J., Garcia, P., Hagglund, T., Dormido, S., Albertos, P., Berenguel, M., 2008. Interactive tool for analysis of time-delay systems with dead-time compensators. Control Engineering Practice 16 (7), 824-835.

He, Y., Wu, M., Liu, G., She, J., 2008. Output feedback stabilization for a discrete-time system with a time-varying delay. IEEE Transactions on Automatic Control 53 (10), 2372-2377.

Ingimundarson, A., Hagglund, T., 2001. Robust tuning procedures of deadtime compensating controllers. Control Engineering Practice 9 (11), 11951208.

Liu, X., Martin, R., Wu, M., Tang, M., 2006. Delay-dependent robust stabilisation of discrete-time systems with time-varying delay. In: Control Theory and Applications, IEE Proceedings-. Vol. 153. IET, pp. 689-702.

Lozano, R., Castillo, P., Garcia, P., Dzul, A., 2004. Robust prediction-based control for unstable delay systems: Application to the yaw control of a mini-helicopter. Automatica 40 (4), 603-612.

Manitius, A. Z., Olbrot, A. W., 1979. Finite spectrum assignment problem for systems with delays. IEEE Transactions on Automatic Control 24 (4), $541-553$.

Meng, X., Lam, J., Du, B., Gao, H., 2010. A delay-partitioning approach to the stability analysis of discrete-time systems. Automatica 46 (3), 610-614.

Mondié, S., Dambrine, M., Santos, O., 2002. Approximation of control laws with distributed delays: a necessary condition for stability. Kybernetika, Praha 38 (5), 541-551.

Normey-Rico, J., Camacho, E., 2008. Dead-time compensators: A survey. Control Engineering Practice 16 (4), 407-428.

Normey-Rico, J., Guzman, J., Dormido, S., Berenguel, M., Camacho, E., 2009. An unified approach for dtc design using interactive tools. Control Engineering Practice 17 (10), 1234-1244.

Normey-Rico, J. E., Camacho, E. F., 2007. Control of Dead-time Processes. Springer-Verlag, London. 
Palmor, Z., 1996. Time-delay compensationsmith predictor and its modifications. The control handbook 1, 224-229.

Pan, Y., Marquez, H., Chen, T., 2006. Stabilization of remote control systems with unknown time varying delays by LMI techniques. International Journal of Control 79 (7), 752-763.

Richard, J., 2003. Time-delay systems: an overview of some recent advances and open problems. Automatica 39 (10), 1667-1694.

Seron, M., Braslavsky, J., Goodwin, G., 1997. Fundamental limitations in filtering and control. [Online] http://www.eng.newcastle.edu.au/ jhb519/book/download.html.

Smith, O., 1957. Closer control of loops with dead time. Chemical Engineering Progress 53 (5), 217-219.

Xie, L., 1996. Output feedback $\mathrm{H}_{\infty}$ control of systems with parameter uncertainty. International Journal of Control 63 (4), 741-750.

Yue, D., Han, Q., 2005. Delayed feedback control of uncertain systems with time-varying input delay. Automatica 41 (2), 233-240.

Zhong, Q., 2004. On distributed delay in linear control laws-part i: Discretedelay implementations. IEEE Transactions on Automatic Control 49 (11), 2074-2080. 


\section{Appendix: Proof of Theorem 1.}

By using the candidate Lyapunov function defined in (13), the increments of $V_{1}$ to $V_{5}$ can be written respectively, as:

$$
\begin{aligned}
\Delta V_{1}(k) & =x_{k+1}^{T} P x_{k+1}-x_{k}^{T} P x_{k} \\
& =\left[A_{0} x_{k}+B_{1} x_{k-h}-B_{1} x_{k-d_{k}}\right]^{T} P\left[A_{0} x_{k}+B_{1} x_{k-h}-B_{1} x_{k-d_{k}}\right]-x_{k}^{T} P x_{k} \\
\Delta V_{2}(k) & =x_{k}^{T} Q x_{k}-x_{k-d_{k}}^{T} Q x_{k-d_{k}}+\sum_{i=k-d_{k+1}+1}^{k-d_{k}} x_{i}^{T} Q x_{i} \\
& \leq x_{k}^{T} Q x_{k}-x_{k-d_{k}}^{T} Q x_{k-d_{k}}+\sum_{i=k-d_{M}+1}^{k-d_{m}} x_{i}^{T} Q x_{i} \\
\Delta V_{3}(k) & =x_{k}^{T}\left(Q_{m}+Q_{M}+Q_{h}\right) x_{k}-x_{k-d_{m}}^{T} Q m x_{k-d_{m}}-x_{k-d_{M}}^{T} Q_{M} x_{k-d_{M}}-x_{k-h}^{T} Q_{h} x_{k-h} \\
\Delta V_{4}(k) & =\delta\left[x_{k}-x_{k-d_{M}}\right]^{T} Q\left[x_{k}-x_{k-d_{M}}\right]-\sum_{i=k-d_{M}+1}^{k-d_{m}} x_{i}^{T} Q x_{i} \\
\Delta V_{5}(k) & =\nu_{k}^{T} Z \nu_{k}-\rho_{1} \sum_{i=k-\rho_{1}}^{k-1} \nu_{i}^{T} Z_{2} \nu_{i}-\left|d_{m}-h\right| \sum_{i=k-\rho_{2}}^{k-\rho_{1}-1} \nu_{i}^{T} Z_{1} \nu_{i}-\sum_{i=k-d_{M}}^{k-d_{k}-1} \nu_{i}^{T} Z_{M} \nu_{i}-\sum_{i=k-d_{k}}^{k-d_{m}-1} \nu_{i}^{T} Z_{M} \nu_{i}
\end{aligned}
$$

where

$$
Z=\rho_{1}^{2} Z_{2}+\left(d_{m}-h\right)^{2} Z_{1}+\delta Z_{M}
$$

and

$$
\delta=d_{M}-d_{m}
$$

Applying the Tchebyschev inequality (Lemma 1 in Guo and Li (2009)) it yields

$$
\begin{gathered}
-\rho_{1} \sum_{i=k-\rho_{1}}^{k-1} \nu_{i}^{T} Z_{2} \nu_{i} \leq-\left[\sum_{i=k-\rho_{1}}^{k-1} \nu_{i}\right]^{T} Z_{2}\left[\sum_{i=k-\rho_{1}}^{k-1} \nu_{i}\right] \\
-\left|d_{m}-h\right| \sum_{i=k-\rho_{2}}^{k-\rho_{1}-1} \nu_{i}^{T} Z_{1} \nu_{i} \leq-\left[\sum_{i=k-\rho_{2}}^{k-\rho_{1}-1} \nu_{i}\right]^{T} Z_{1}\left[\sum_{i=k-\rho_{2}}^{k-\rho_{1}-1} \nu_{i}\right]
\end{gathered}
$$


and so on

$$
\begin{aligned}
& \Delta V_{5}(k) \leq \nu_{k}^{T} Z \nu_{k}-\left[x_{k}-x_{k-\rho_{1}}\right]^{T} Z_{2}\left[x_{k}-x_{k-\rho_{1}}\right]- \\
& \quad\left[x_{k-\rho_{1}}-x_{k-\rho_{2}}\right]^{T} Z_{1}\left[x_{k-\rho_{1}}-x_{k-\rho_{2}}\right]-\sum_{i=k-d_{M}}^{k-d_{k}-1} \nu_{i}^{T} Z_{M} \nu_{i}-\sum_{i=k-d_{k}}^{k-d_{m}-1} \nu_{i}^{T} Z_{M} \nu_{i}
\end{aligned}
$$

For some matrices defined as $\tilde{S}=\left(\begin{array}{lllll}0 & S_{1}^{T} & S_{2}^{T} & 0 & 0\end{array}\right)^{T}, \tilde{T}=\left(\begin{array}{lllll}0 & T_{1}^{T} & 0 & T_{2}^{T} & 0\end{array}\right)^{T}$ with appropriate dimensions, it yields

$$
\begin{aligned}
0= & 2 \lambda_{k}^{T} \tilde{S}\left[x_{k-d_{m}}-x_{k-d_{k}}-\sum_{i=k-d_{k}}^{k-d_{m}-1} \nu_{i}\right] \leq \\
& 2 \lambda_{k}^{T} \tilde{S}\left[x_{k-d_{m}}-x_{k-d_{k}}\right]+\left(d_{k}-d_{m}\right) \lambda_{k}^{T} \tilde{S} Z_{M}^{-1} \tilde{S}^{T} \lambda_{k}+\sum_{i=k-d_{k}}^{k-d_{m}-1} \nu_{i}^{T} Z_{M} \nu_{i} \\
0= & 2 \lambda_{k}^{T} \tilde{T}\left[x_{k-d_{k}}-x_{k-d_{M}}-\sum_{i=k-d_{M}}^{k-d_{k}-1} \nu_{i}\right] \leq \\
& 2 \lambda_{k}^{T} \tilde{T}\left[x_{k-d_{k}}-x_{k-d_{M}}\right]+\left(d_{M}-d_{k}\right) \lambda_{k}^{T} \tilde{T} Z_{M}^{-1} \tilde{T}^{T} \lambda_{k}+\sum_{i=k-d_{M}}^{k-d_{k}-1} \nu_{i}^{T} Z_{M} \nu_{i}
\end{aligned}
$$

Finally, the forward difference $\Delta V(k)$ can be put as

$$
\Delta V(k) \leq \lambda_{k}^{T} \Omega \lambda_{k}-\sum_{i=k-d_{k}}^{k-d_{m}-1} \nu_{i}^{T} Z_{M} \nu_{i}-\sum_{i=k-d_{M}}^{k-d_{k}-1} \nu_{i}^{T} Z_{M} \nu_{i}<0
$$

where the augmented state is defined as

$$
\lambda_{k}^{T}=\left(\begin{array}{lllll}
x_{k} & x_{k-d_{k}} & x_{k-d_{m}} & x_{k-d_{M}} & x_{k-h}
\end{array}\right) .
$$

and the matrix $\Omega$ is

$$
\Omega=\left(\begin{array}{ccccc}
\Omega_{1} & \Omega_{2} & \Omega_{3} & 0 & \Omega_{4} \\
(*) & \Omega_{5} & S_{1}-S_{2}^{T} & -T_{1}+T_{2}^{T} & \Omega_{6} \\
(*) & (*) & \Omega_{7} & 0 & Z_{1} \\
(*) & (*) & (*) & \Omega_{8} & 0 \\
(*) & (*) & (*) & (*) & \Omega_{9}
\end{array}\right)
$$


with

$\Omega_{1}=A_{0}^{T} P A_{0}+\left(A_{0}-I\right)^{T} Z\left(A_{0}-I\right)-P+(\delta+1) Q+Q_{M}+Q_{m}+Q_{h}-Z_{2}$

$\Omega_{2}=-A_{0}^{T} P B_{1}-\left(A_{0}-I\right)^{T} Z B_{1}$

$\Omega_{3}= \begin{cases}0 & h \leq d_{m} \\ Z_{1} & h>d_{m}\end{cases}$

$\Omega_{4}=A_{0}^{T} P B_{1}+\left(A_{0}-I\right)^{T} Z B_{1}+ \begin{cases}Z_{2} & h \leq d_{m} \\ 0 & h>d_{m}\end{cases}$

$\Omega_{5}=B_{1}^{T} P B_{1}+B_{1}^{T} Z B_{1}-Q$

$\Omega_{6}=-B_{1}^{T} P B_{1}-B_{1}^{T} Z B_{1}$

$\Omega_{7}=-Q_{m}-Z_{1}+ \begin{cases}0 & h \leq d_{m} \\ -Z_{2} & h>d_{m}\end{cases}$

$\Omega_{8}=-Q_{M}$

$\Omega_{9}=B_{1}^{T} P B_{1}+B_{1}^{T} Z B_{1}-Q_{h}-Z_{1}+ \begin{cases}-Z_{2} & h \leq d_{m} \\ 0 & h>d_{m}\end{cases}$

Then, after some lengthy algebraic manipulations and Schur complement, the inequality (15) is obtained.

Following the baseline of reasoning in Guo and Li (2009), the LMIs in (16)-(17) are obtained and the final result (12) is achieved. 